\title{
ERYTHEMA NODOSUM ASSOCIATED WITH STAPHYLOCOCCUS SPECIES INFECTION IN A CHILD
}

\author{
Sanja Knezevic ${ }^{1,2}$, Tijana Prodanovic ${ }^{2}$, Marija Radovanovic ${ }^{1,2}$, Nikola Prodanovic ${ }^{3,4}$ and Gordana Kostic ${ }^{1,2}$ \\ ${ }^{I}$ University of Kragujevac, Faculty of Medical Sciences, Department of Pediatrics, Kragujevac, Serbia \\ ${ }^{2}$ Clinical Center Kragujevac, Pediatric Clinic, Kragujevac, Serbia \\ ${ }^{3}$ Clinical Center Kragujevac, Orthopedics and Traumatology Clinic, Kragujevac, Serbia \\ ${ }^{4}$ University of Kragujevac, Faculty of Medical Sciences, Department of Surgery, Kragujevac, Serbia
}

\section{Corresponding author:}

\section{Sanja Knezevic}

19b/3 Kralja Milana IV Street, 34000 Kragujevac, Srbija

Phone: +381(0) 6912190

E-mail: sanjaknez1980@yahoo.com

\begin{abstract}
Erythema nodosum (EN) is a poly-etiological disease with an acute flow that is characterized by symmetric emergence of painful nodules often in pretibial areas. A twenty-month-old male child was admitted to hospital for evaluation of the eruptive skin changes in the lower extremities and forearms. The disease began 10 days before getting febricity and loose stools. The laboratory analysis showed an elevated erythrocyte sedimentation rate and leukocytosis. Blood cultures demonstrated the presence of coagulase-negative Staphylococcus, while Proteus vulgaris was isolated in urine cultures. After initiation of the empiric antibiotic therapy and then, the targeted antibiotic therapy according to the antibiogram, there was a significant improvement in a general condition and regression of cutaneous lesions. Erythema nodosum in the present case, is the result of staphylococcal bacteremia although Proteus vulgaris cannot be excluded as a cause.
\end{abstract}

Keywords: Erythema nodosum, Bacteremia, Staphylococcus.

\section{sciendo}

\author{
UDK: $616.511-053.2$
}

$616.98: 579.86$

Eabr 2022; 23(4):369-372

DOI: $10.2478 /$ sjecr-2020-0017 


\section{INTRODUCTION}

Erythema nodosum (EN) is a poly-etiological disease with an acute flow that is characterized by symmetric emergence of painful nodules often in pretibial areas. The prevalence in adults is $1-5$ per 100,000 while in children, it has not been established. It occurs most often between 20 and 40 years of age and is more common in women (1). It usually occurs in children between the ages of 8 and 10 and is equally present in both sexes (2). According to the etiology, EN may be primary (idiopathic) or secondary. Each EN for which the secondary etiology has not been proven, is defined as idiopathic. In most cases of children with EN, this disease is idiopathic (3). The most common causes of the secondary EN are infectious agents (streptococcal infection, Mycobacterium tuberculosis, viruses, Mycoplasma pneumoniae, parasites, fungi) and drugs (sulfonamides, amoxicillin, contraceptives). In $3-10 \%$ of cases, the first manifestation of malignancy (leukemia, non-Hodgkin lymphoma, rarely, myeloid leukemia) is EN. In the systemic autoimmune diseases (sarcoidosis, SLE), EN occurs in $11-25 \%(4,5)$. It can also occur as a part of other diseases (Crohn's disease, ulcerative colitis, M. Behcet, cat-scratch disease). In addition, there was a case of EN in children caused by Shigella flexneri (6). Also, the cases of EN are well described in a normal pregnancy.

In general, erythema nodosum represents a ductile structure inflammation of the subcutaneous tissue, septal panniculitis, followed by the eruptive changes on the skin. The changes are localized in hypodermis, but without vasculitis. The onset of the disease is usually manifested by fever, arthralgia, myalgia, the upper respiratory tract infection and abdominal pain. The pathognomonic signs of erythematous are subcutaneous nodules that are painful on palpation, sensitive and symmetrically localized in the front of the lower extremities (4). The indicated changes are not ulcers and they are usually withdrawn after 2-8 weeks without leaving scars on the skin. Sometimes, EN is the first clinical manifestation of the disease for which the diagnosis requires a multidisciplinary approach.

\section{CASE REPORT}

A 20-month-old male child is brought to hospital for examination because of the eruptive skin changes, initially on the shins and then on the forearms. The disease began 10 days before getting the fever up to $38.7^{\circ} \mathrm{C}$ (the highest 39 $\left.{ }^{\circ} \mathrm{C}\right)$. Along with the increase in body temperature, rare fluid stools occurred (3-4 per day, on the day of the admission, 10 stools) and he was treated with probiotics. The day before the admission, the skin changes occurred. During the physical examination, the child was conscious, with fever $\left(38^{\circ} \mathrm{C}\right)$, eupneic, eutrophic. The skin of the anterolateral side of both shins was pale, with less elasticity and turgor and with present erythematous nodules, painful and warm on palpation
(Figure 1A, 1B). A pair of similar skin lesions was present on both forearms (Figure 1C).
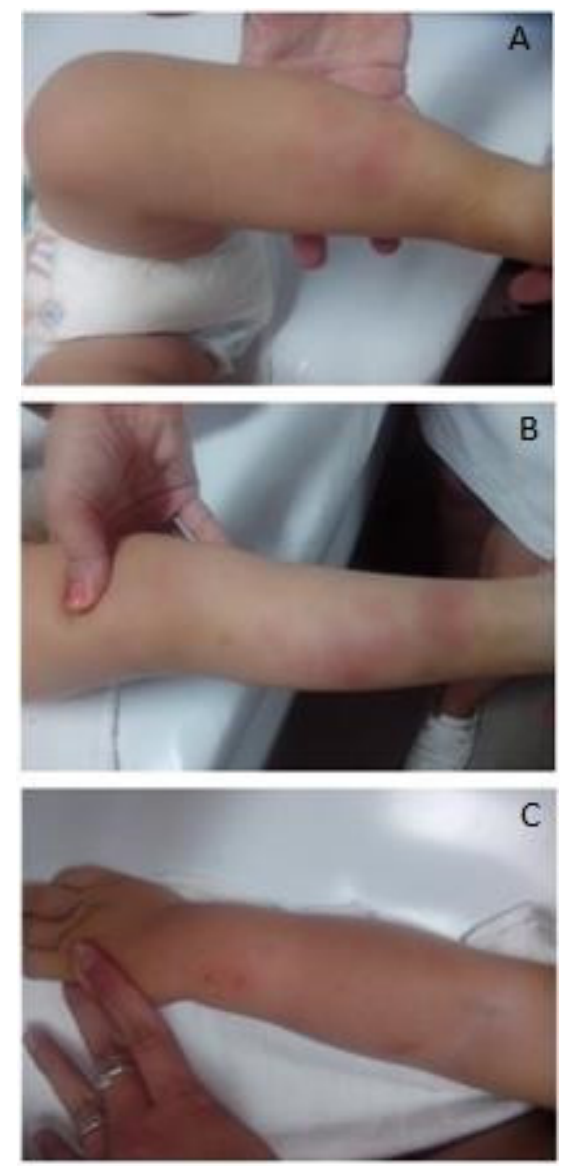

Figure 1.

The laboratory examination showed leukocytosis $14.0 \times 10^{9} / \mathrm{L}$ with neutrophilia $50.8 \%$. The erythrocyte sedimentation rate (ESR) was elevated $(45 \mathrm{~mm} / \mathrm{hr})$. The other indicators of inflammation were not increased (C-reactive protein was 3.9 , procalcitonin was 0.120 ). The erythrocyte and platelets count as well as hemoglobin concentration were in normal range. The urinary analysis showed no pyuria but the urine cultures for bacteria and fungi showed Proteus vulgaris $(100000 \mathrm{cfu} / \mathrm{ml})$. The nasal and pharyngal smear showed negative results. The blood cultures for bacteria and fungi showed coagulase-negative Staphylococcus species (MRSA). There were no isolated pathogenic bacteria in feces. The Rotagen test showed negative results. AntiStreptococcal (ASO) titer was normal. The serological analysis (Immunoglobulin M) of Mycoplasma pneumonia was negative. The chest radiograph showed no hilar adenopathy or pulmonary infiltrates. On the basis of the clinical presentation, medical history and laboratory indicators, the boy was diagnosed with: Erythema nodosum; Sepsis. The treatment was initiated by a parenteral administration of a broad spectrum of antibiotics 
(cephalosporin's $3^{\text {rd }}$ generation) and after obtaining the blood cultures findings, the anti-staphylococcal drug (Vancomycin) was administered. The therapy led to the improvement of clinical symptoms described by the regression of skin lesions and afebrility. After ten days of the therapy, the skin lesions were repaired without occurrence of ulceration and scarring and with normalization of the laboratory parameters (Figure 2).

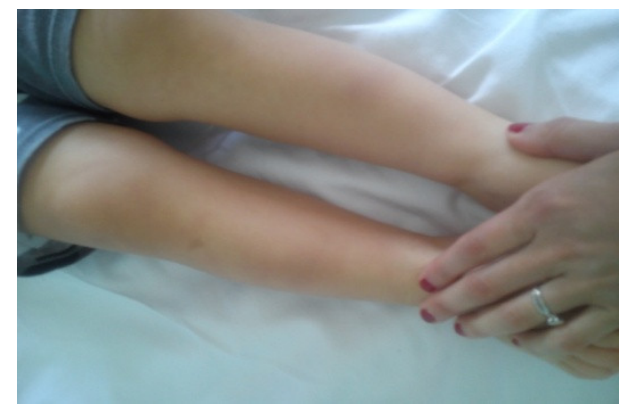

Figure 2.

\section{DISCUSSION}

In this study, a less typical case of EN for the age is described, as well as causes and localization of the reported changes. Most of the published studies show that the most common age for EN is 8-10 years whereas in our case, it was quite early, in the 20th month of life $(2,4)$. This was the earliest detection of EN in our clinic in the last couple of years. Although many studies have shown that the most common cause of EN in children is beta hemolytic streptococcus group A (50-77\%), in our case, the cause was Staphylococcus species. Taking into consideration that the patient had frequent loose stools, the Rotagen stool culture test was made and the result was negative. Often, a gram-negative bacillus Yersinia enterocolitica, as the cause of acute diarrhea with occurrence of the abdominal pain, is associated with EN. Also, salmonella infection can be the cause of erythema nodosum. These two possible causes were not found in feces, and the stools were normalized by the antibiotic treatment. Although the patient did not have signs of the respiratory infections, the lung radiological examinations and serological analysis excluded pneumonia caused by Mycoplasma pneumoniae (as an atypical cause that can lead to EN) (7). Since BCG scar was clearly visible (the child was vaccinated at birth), there was no evidence of tuberculosis infection in the family, the chest X-rays were normal and there was no need for PpD3 (Protein phosphatase D3) test. Moreover, the hypersensitive reaction of subcutaneous tissue to the presence of mycobacterium tuberculosis is manifested in the form of subcutaneous nodules (localized mainly on the back side of the lower extremities), which are prone to ulceration and scarring after the rehabilitation process. Since the common cause of EN is an infectious agent, the laboratory diagnosis usually showed the elevated parameters of inflammation. In the study of Litwin and coworkers, ten of total twelve children had the elevated CRP values, while two children had the CRP value below $5 \mathrm{mg} / \mathrm{L}$ (8). In $75 \%$ of children with $\mathrm{EN}$, the diagnosis can be set on the basis of a medical history of patient, laboratory tests and clinical symptoms (2). In patients with an undefined etiological cause or atypical forms of EN, the diagnostic procedure should be extended for the detection of serious diseases, such as systemic lupus erythematosus (SLE), Crohn's disease and ulcerative colitis (3). Also, EN could be a very rare manifestation of Behcet disease and sarcoidosis in children (9). Based on the clinical presentation, laboratory and other analyses and due to staphylococcus that was isolated in the blood cultures, the established diagnosis was

erythema nodosum probably caused by infection of staphylococcus. Since the urine cultures showed Proteus vulgaris infection, it is possible that both agents caused occurrenceof $\mathrm{EN}$. The literature describes the so-called double diagnosis that caused EN in children, such as streptococcal infections plus mycoplasma pneumonia or streptococcal infection plus latent tuberculosis infection or streptococcal infection plus chlamydia pneumonia. However, only $56.4 \%$ children had a confirmed infectious agent as the cause of EN (10). Very often, EN represents the first manifestation of tuberculosis infection (11). Staphylococcal septicemia and subcutaneous inflammatory nodules are well described in patients with multiple brain abscesses (12). The first case of EN associated with coagulase-negative staphylococcus infection was described in 2012, in young women (13). Our case represents the first described case of EN in children caused by staphylococcus infection.

The predicative sites for the formation of skin lesions in EN are pretibial regions of the shins (14) as shown in Figure $1 \mathrm{~A}$ and $1 \mathrm{~B}$. The skin lesions are most commonly localized on legs bilaterally, unilaterally and very rare on hands (15). In our 20-month-old patient, the changes were localized in addition to the shins and forearms, especially around the wrists. In the differential diagnosis of EN, in children, the allergic skin diseases, infections, insect bites, trauma and many other conditions should be excluded. We need to look for the most common infectious causes that provoked EN as well as for those less frequent. When there was EN with an unknown trigger, a possible connection with other diseases should be taken into consideration and in this sense, further investigations should be performed. As the infectious agent in children is the most common cause of the disease, the laboratory tests of blood, urine and bacteriological cultures are conducted in order to find the possible cause. The elevated inflammatory parameters, ESR and leukocyte count with neutrophilia showed a bacterial infection. The general condition of the child was in this favor. We got the confirmation that in the blood cultures, Staphylococcus was isolated and Proteus, in the urine culture. Because the disease represents the hypersensitive reaction of the subcutaneous tissue structure of the infectious agent in the twentymonth-old boy, the final diagnosis was erythema nodosum. By giving the antibiotic therapy, at the beginning, the initial broad-spectrum antibiotic and targeted antibiotic, with the symptomatic treatment, there was a rehabilitation of the skin lesions with clinical recovery and normalization of the 
laboratory parameters. After two weeks of the treatment, there was a complete recovery.

\section{CONCLUSION}

Erythema nodosum is a rare infectious disease, atypically associated with coagulase - negative staphylococcus, especially in young children. The microbial spectrum of EN may be much greater than we know, since all microbes can potentially induce the activation of immunological events in their host through complex mechanisms.

\section{ETHICS APPROVAL AND CONSENT TO PARTICIPATE}

The study was conducted in accordance with the ethical standards of the committee responsible for human experimentation (institutional and national) and the Helsinki Declaration of 1975 , as revised in 2013. Voluntary written and informed consent was obtained from the patient prior to enrollment in the study

\section{CONFLICT OF INTEREST}

There are no conflicts of interest.

\section{FUNDING}

None.

\section{REFERENCES}

1. Habif, T. (2009). Hypersensitivity syndromes and vasculitis. Clinical Dermatology (5th ed.). Philadelphia: Mosby Elsevier.

2. Kakourou T, Drosatou P, Psychou F, Aroni K, Nicolaidou P. Erythema nodosum in children: a prospective study. J Am Acad Dermatol 2009; 44: 17-21.

3. Kliegman, R., Nelson, WE. (2011). Panniculitis and erythema nodosum. Nelson Textbook of Pediatrics (19th ed.). Philadelphia: Elsevier Saunders.

4. Schwartz AR, Nervi S. Erythema nodosum: A Sign of Systematic Disease. J Am Acad Fam Physicians 2007; 75: 695-700.

5. Mañá J, Marcoval J. Skin manifestations of sarcoidosis. Presse Med 2012; 41: 355-374.

6. Chowanies M, Starba A, Wiland P. Erythema nodosum - review of the literature. Reumatologya 2016; 54(2): 7982.

7. Trčko K, Marko PB, Miljković J. Leukocytoclastic vasculitis induced by Mycoplasma pneumoniae infection. Acta Dermatovenero Croat 2012; 20: 118-121.

8. Lytwin L. Machura E. The etiology and clinical manifestation of erythema nodosum in hospitalized children analysis of 12 cases. Preliminary report. Dev Period Med 2014; 18: 506-512.

9. Atmaca L, Boyvat A, Yalçındağ FN, Atmaca-Sonmez P, Gurler A. Behçet disease in children. Ocul Immunol Inflamm 2011; 19: 103-107.

10. Aydın-Teke T, Tanır G, Bayhan GI, Metin O, Oz N. Erythema nodosum in children: evaluation of 39 patients. Turk J Pediatr 2014; 56: 144-149.

11. Whig J, Mahajan V, Kashyap A, Gupta S. Erythema nodosum: atypical presentation of common disease. Lung India 2010; 27: 181-182.

12. Satoshi Y, Kiminobu Y, Ryoko S, Makoto T, Makoto U. Staphylococcus cohnii as a cause of multiple brain abscesses in Weber-Christian disease. J Neurol Sci 2015; 238: 97-100.

13. Giordano N, Corallo C, Miracco C, et al. Erythema nodosum associated with Staphylococcus xylosus septicemia. J Microbiol Immunol Infect 2016; 49: 134 -137.

14. Celebi S, Hacimustafaglu M, Yuceer M.B, Aygun F.D, Yenigul C. Erythema nodosum in children. J of Pediatric Infect 2011; 5(4): 136-140.

15. Mert A, Kumbasar H, Ozaras R, et al. Erythema nodosum: an evaluation of 100 cases. Clin Exp Rheumatol 2007; 25: 563-570. 